Cultures \& Conflits

03 | automne 1991

Mafia, drogue et politique

\title{
Le trafic de drogue au Pakistan : une affaire d'Etat?
}

\author{
Alain Labrousse
}

\section{(2) OpenEdition}

Journals

Édition électronique

URL : http://journals.openedition.org/conflits/113

DOI : 10.4000/conflits.113

ISSN : $1777-5345$

Éditeur :

CCLS - Centre d'études sur les conflits lilberté et sécurité, L'Harmattan

Édition imprimée

Date de publication : 17 octobre 1991

ISSN : 1157-996X

Référence électronique

Alain Labrousse, "Le trafic de drogue au Pakistan : une affaire d'Etat ? ", Cultures \& Conflits [En ligne], 03 | automne 1991, mis en ligne le 31 décembre 2002, consulté le 30 mars 2021. URL : http:// journals.openedition.org/conflits/113; DOI : https://doi.org/10.4000/conflits.113

Ce document a été généré automatiquement le 30 mars 2021

Creative Commons License 


\title{
Le trafic de drogue au Pakistan : une affaire d'Etat?
}

\author{
Alain Labrousse
}

1 Chaque année 100 tonnes d'héroïne, après avoir traversé le Pakistan du nord au sud, sont acheminées en Europe par les voies maritimes, aériennes et surtout empruntent les routes des Balkans via l'Iran et la Turquie(1). 50 tonnes sont encore destinées au million d'héroïnomanes que compte le pays et dont le nombre ne cesse d'augmenter. Ce marché représente au niveau de la vente au détail dans les pays européens une vingtaine de milliards de dollars, dont 2 à 4 retournent effectivement dans le pays(2). Il suppose des réseaux nombreux et bien organisés. Les risques encourus, par ceux qui tentent de lever un coin du voile au Pakistan, ajoutés au peu d'intérêt porté par les chercheurs et les journalistes occidentaux au pays qui est pourtant le principal responsables de la toxico-manie chez nous, font que les organisations criminelles du Pakistan et leur lien avec le monde politique n'ont pas été étudiés(3). L'histoire de la spécialisation récente du Pakistan dans ces activités peut pourtant fournir un certain nombre de pistes à la recherche.

Les événements de l'année 1979 dans le Croissant d'or

2 Cette spécialisation a été provoquée par la rencontre, dans une certaine mesure fortuite, d'une politique prohibitionniste mise en lace en 1979 par la dictature du général Zia et d'événements politiques et militaires qui ont ébranlé la réunion du Croissant $d$ or à partir de cette même année(4).

3 A cette date le pays comptait quelque 500000 consommateurs d'opium et les hakims (guérisseurs), au nombre de 50 000, continuaient à le prescrire pour guérir insomnie, diarrhée, dysenterie, diabète, rhumatisme, toux et troubles digestifs chez l'enfant. Bien que l'Etat ait exercé un contrôle sur la culture et les ventes de la production d'opium afin qu'il ne soit pas transformé en héroïne, une partie de la récolte était clandestinement exportée en Iran pour les besoins des toxicomanes.

4 Avec la décision de transformer le pays en république islamique prise par la dictature militaire du général Zia en 1979, la consommation de l'opium - comme celles du haschisch et de l'alcool - ont été interdites. La loi prévoyait pour le trafic de ces 
drogues, sans faire de distinction entre elles, cinq ans d'emprisonnement, trente coups de fouets et une amende. En 1983 la peine de prison fut augmentée jusqu'à 25 ans pour la possession de plus de dix grammes d'héroïne et d'un kilo d'opium en vue du trafic(5).

En ratifiant en 1978 son adhésion à la Convention unique sur les stupéfiants des Nations unies le Pakistan, qui s'était également engagé à mettre fin aux cultures de pavot, annonça qu'il serait interdit de semer à la fin de l'année 1978. Les paysans profitèrent de ce répit pour réaliser une production record qui passa de 500 tonnes en 1978 à 800 tonnes au printemps 1979.

Comme la nouvelle loi - Amendement Hadd - ordonnait la fermeture des 330 points de vente d'opium dans le pays, les trafiquants projetèrent d'exporter la plus grande partie de cette production en Iran où il existait un million d'opiomanes et près de 100000 héroïnomanes. Mais la révolution islamique qui éclata dans ce pays en janvier 1979, désorganisa les circuits et affecta la consommation leur laissant sur les bras des stocks considérables.

7 Or, le hasard fit que la récolte dans le Triangle d'or, la première région productrice d'opium dans le monde, fut gravement affectée par la sécheresse et passa de 500/600 tonnes à 200 tonnes(6). Une aubaine pour les trafiquants pakistanais, seuls capables de répondre à un marché affamé d'opiacés : "Il ne leur restait plus qu'à transformer leur opium en héroïne. On suppose que des chimistes venus de France, de HongKong et d'Iran, appelés à la rescousse, firent partager aux Pathans leur savoir-faire. C'est ainsi que débuta l'histoire de la production d'héroïne au Pakistan et en Afghanistan"(7).

8 Mais l'année 1979, décidément fertile en événements, n'était pas terminée que les Russes envahissaient, en décembre, l'Afghanistan. Cet événement allait d'une part refouler le trafic de l'Est - c'est-à-dire le Pakistan - et en envoyant des millions de réfugiés afghans dans les zones tribales, les transformer en plaque tournante du trafic d'armes et de drogue. Ces zones tribales sont situées dans une des quatre grandes régions du Pakistan, la Province Frontière du Nord-Ouest (NWFP) qui a été détachée du Penjab par les Britanniques en 1901. Elle est à son tour divisée en trois entités administratives distinctes: les districts intégrés (settled areas) - 6,2 millions d'habitants en 1985 - où s'appliquent pleinement les lois de l'Etat Pakistanais et deux zones de juridiction spéciales les FATA et PATA.

9 Les FATA(8) (agences tribales administrées par le gouvernement fédéral.) sont au nombre de six et regroupent 2,1 millions d'habitants dont la population appartient à l'ethnie pathane(9). Leur particularité, qui explique en particulier l'importance qu'y ont pris la production et le trafic de drogues, est que les lois civiles et criminelles de l'État pakistanais ne s'y appliquent pas. Cette situation est une conséquence du traité de Gandamak, imposé en 1879 par les Anglais au gouvernement afghan afin de créer une zone tampon face à la menace de la Russie tsariste(10). La cession des territoires pathans de Peshawar au Pakistan devint effective en 1893. Les Britanniques s'attribuèrent la souveraineté sur toutes les régions situées au sud de la ligne de crête des monts Souleiman. Cette frontière imaginaire qui coupa en deux, non seulement l'ethnie pathane, mais les tribus elles-mêmes, fut nommée la Ligne Durand du nom d'un secrétaire aux Affaires étrangères. Après avoir vainement essayé de soumettre les Pathans par la force au prix de pertes considérables(11), les Anglais préférèrent leur accorder un statut spécial d'autonomie dont a hérité l'État pakistanais au moment de l'Indépendance en 1947. Les Afghans, qui appartiennent en majorité à l'ethnie pathane, 
n'ont, par contre, jamais admis cette division et appuient la formation d'un État indépendant, le Pashtounistan, qui serait bien entendu leur allié(12).

Si dans les zones tribales le gouvernement du Pakistan garde la souveraineté sur les axes routiers, les voies de chemins de fer et les casernes, les seules autorités sont les Jirgas, conseils des barbes blanches, censés appliquer les lois islamiques. Le résultat c'est qu'un méfait commis à plus de cinq mètres de la route - assassinat, passage de marchandises ou de drogue en contrebande - ne peut entraîner l'intervention des forces de l'ordre. Aussi estime-t-on aujourd'hui à 7000 le nombre des délinquants qui seraient réfugiés dans les zones tribales pakistanaises d'où il leur est possible, en cas de nécessité de passer en Afghanistan : la notion de frontière ayant peu de réalité pour ces tribus qui appartiennent toutes à l'ethnie pathane.

11 C'est précisément dans les régions à cheval sur la frontière mais surtout du côté pakistanais plus sûr, qu'ont été installés les premiers laboratoires de morphine base en 1975 et que se trouvent aujourd'hui les 150 à 200 laboratoires d'héroïne - au sujet desquels il vaudrait mieux d'ailleurs utiliser l'expression de "cuisines volantes" (makeshifts) - recensées par les services antidrogue américains(13). Ils sont contrôlés par ce que l'ont pourrait appeler des organisations criminelles qui épousent la structure et le type de relations sociales existant dans les tribus, permettant l'ascension sociale et politique de trafiquants, tel Haji Ayub Zakakhel Afridi.

Organisations tribales et trafic de drogue

12 Il n'est pas impossible de visiter, comme nous l'avons fait, son palais, situé peu avant le bourg de Landikotal, au bord de la grand qui mène à la frontière afghane(14). Haji Ayub est un camionneur de la tribu des Afridi, une des plus importantes chez les Pathans, qui a fait fortune dans le commerce du haschisch, puis de d'héroïne. Un mandat d'amener a été lancé en 1983 contre lui à la suite de la découverte de 17 tonnes de haschisch dans un entrepôt du Baloutchistan. Il a été condamné à un an de prison pour ne s'être pas présenté devant le tribunal. Trois ans plus tard, il a fait objet d'un nouvel avis de recherche à la suite de l'arrestation d'un passeur en Belgique qui l'a dénoncé comme son fournisseur. Ce mandat d'amener a été renouvelé en 1989, sous le gouvernement de Bénazir Bhutto. Lorsque les autorités ont parlé de faire raser son palais par des bulldozers, des centaines de guerriers afridi, armés de kalachnikovs et de bazookas, sont descendus de la montagne pour prendre la défense de celui qui est devenu à leurs yeux un des symboles de la réussite sociale. Comme toute action militaire du gouvernement central dans ces régions, dont les habitants sont traditionnellement des contrebandiers et des guerriers, entrainerait des combats meurtriers, et, à terme, des menées sécessionnistes appuyées par l'Afghanistan, le gouvernement de Benazir a reculé. Et maintenant, Haji Ayub siège sur les bancs de l'Assemblée nationale dans les rangs du parti au pouvoir... en jouissant de l'immunité parlementaire.

A l'ombre de ce cas exemplaire, les grandes familles pathanes de l'héroïne ne seraient pas moins d'une quarantaine sur les quelques cinquante grandes organisations criminelles de la drogue du Pakistan. Il convient de distinguer parmi elles celles dont l'assise - à l'exemple d'Haji Ayub - reste essentiellement rurale et tribale (et qui se sont enrichies en passant des caravanes de mules chargées de cotonnades et d'appareils électroménagers aux camions qui transportent du haschisch et de l'héroïne), des parvenus à qui il faudra, à l'instar de leurs homologues colombiens, plusieurs générations pour entrer dans le cercle fermé des élites urbaines pakistanaises(15). Si ces organisations sont capables d'acheminer la drogue des zones tribales jusqu'aux 
lieux d'embarquements ou jusqu'aux frontières du pays, elles n'ont probablement pas les contacts internationaux qui leur permettraient d'avoir une participation à son exportation. L'une de ces familles possède néanmoins le journal le plus influent de la province, dans les colonnes duquel on ne manque jamais de vitupérer contre les trafiquants de drogue, "marchands de morts".

D'autres étaient déjà riches et influentes avant le boom de l'héroïne. Il leur a simplement fourni un moyen supplémentaire de disputer le pouvoir aux élites Penjabi, qui occupent traditionnellement une position dominante au Pakistan. Elles possèdent en général des établissements industriels et commerciaux dans tout le pays et ont des représentants dans l'armée, le gouvernement et le personnel diplomatique. Leurs enfants étudient en Angleterre ou aux Etats-Unis. Elles ont donc accès au marché international. Le fonctionnement des activités criminelles de ces familles, qui se superpose à celui de leurs activités légales, n'est pas fondamentalement différent de celui des mafias urbaines qui repose également sur des liens de parenté. Mais l'appartenance tribale leur assure des réseaux plus étendues et sans doute bénéficiant d'une plus grande étanchéité.

La structures de ces organisations a été décrite de la façon suivante par l'un de mes informateurs : "Au sommet de la pyramide, un homme d'affaire millionnaire qui a des contacts très étroits avec des hommes politiques, des hauts fonctionnaires, des responsables de compagnies de transport maritimes ou aériennes. Les membres de sa famille élargie sont associés à l'affaire sous le nom de "contrôleurs", et ce sont eux qui ont affaire au deuxième cercle. Ils recrutent leurs propres parents qui sont les exécuteurs chargés de convoyer la drogue et des autres opérations. Les liens familiaux garantissent que personne ne parlera. On trouve beaucoup d'étrangers dans le deuxième cercle composé de chimistes et de courriers. L'organisation en cellules et l'étanchéité de ces dernières font que personne ne connaît l'ensemble du réseau (16). Les mafias tribales ne sont pas les seules à convoyer de la drogue, une mafia militaire, beaucoup plus discrète, s'est aussi constituée afin d'enrichir ceux qui disposent de moyens de coercition.

La guerre en Afghanistan et la mafia militaire

Si en dix ans le Pakistan est devenu le deuxième producteur mondial d'héroïne après la Birmanie, il le doit en grande partie à la guerre qui s'est déroulée en Afghanistan(17). Dans ce pays l'absence de contrôle du territoire de la part de l'État, les conséquences des bombardements soviétiques sur les zones agricoles les plus riches, Ies besoins en armes des différentes factions de la résistances à mesure que les livraisons de leurs protecteurs se sont faîtes plus parcimonieuses, ont provoqué une extension considérable de la production d'opium. La plus grande partie de celle-ci est maintenant "exportée" au Pakistan pour être transformée en héroïne. La place de l'héroïne du Pakistan, deuxième producteur mondial, ne résulte cependant pas d'une simple contamination, ou de l'existence de régions mal contrôlées comme les agences tribales, mais bien d'une part active prise par certains services relevant de l'appareil d'État.

Dès le début du conflit les services secrets de l'armée pakistanaise, Inter Service Intelligence (ISI) se sont vu confer par les Etats-Unis le monopole de la livraison de leur aide financière et militaire à la Résistance afghane. Cela a eu des conséquences politiques importantes dans la mesure où ils ont favorisé les secteurs les plus intégristes de la résistance(18), et ils ont également profité de cette position de monopole pour prendre une part importante dans le trafic d'héroïne. La presse 
pakistanaise a pris le risque, sous la dictature du général Zia, de démontrer comment les camions scellés NLC, qui livraient des armes aux Moudjahidins, rapportaient d'Afghanistan de l'opium transformé dans les zones tribales, puis de l'héroïne qui était ensuite expédiée dans le sud du pays afin d être exportée. Il est tout à fait impossible que la CIA, qui travaillait en étroite collaboration avec l'ISI, n'ait pas été au courant de ces trafics. Certains observateurs vont plus loin : ils affirment qu'elle l'a couvert(19). Un correspondant de presse étranger, qui pour des raisons de sécurité n'a pas publié son enquête, a pu établir que les missiles Stinger, qui ont fait perdre aux Soviétiques et aux gouvernementaux la maîtrise du ciel en 1987, avaient été livrés en priorité dans les zones où se trouvaient les plus importantes productions de pavot. Les représentants de la DEA à Peshawar ne se privent pas de dire aujourd'hui, en privé, que la CIA est responsable d'une situation qu'on leur demande de régler.

Il semble bien qu'une grande partie de ce trafic n'était pas destiné seulement à l'enrichissement personnel de certains militaires mais constituait, et constitue encore dans une large mesure, une prise en main par l'État des ressources fournies par la drogue. Le général Zia a incontestablement couvert la mise en place de cette connexion militaire, qui ne représentait pas, loin s'en faut toute l'armée, mais une clique extrêmement puissante. Sur son organisation et son fonctionnement on en est réduit aux conjectures. Il est vraisemblable qu'elle recouvre les modes de fonctionnement des loges militaires à l'intérieur desquelles la hiérarchie est respectée, mais où ce sont les subordonnés qui prennent le plus de risques. Au cours d'un grand procès provoqué par les découvertes d'incorruptibles de la police norvégienne, le général Fazle Haq homme de confiance du général Zia et gouverneur de la Province du Nord(20) a été impliqué. Les services antidrogue du gouvernement de Bénazir Bhutto et la presse américaine ont confirmé ces dénonciations, mais sans que des preuves décisives soient jamais apportées. En revanche le pilote privé du chef de l'État, le major Farouq Hamid, qui était la pièce maîtresse d'un grand trafic international d'héroïne, n'a été arrêté et condamné qu'en 1989, c'est-à-dire après la disparition de son protecteur. Par ailleurs, un des aides de camp du chef de l'Etat tenta, en 1981, de passer de l'héroïne dans un avion qui amenait Zia à New York où il devait s'adresser à blée générale de l'ONU. Il fut découvert, chassé de l'armée, chef de l'État lui fit attribuer un poste dans la fonction publique. Ce dernier usa également de son pouvoir pour faire relâcher Abdullah Bhatti, un des grands caïds de la drogue. Enfin, toujours dans l'affaire de la "connexion norvégienne" que nous avons évoquée, lorsque le blanchisseur de l'argent du réseau, le banquier Hamid Hasnain, fut arrêté, on trouva sur lui les chéquiers et des relevés bancaires du président et de son épouse. Cette dernière tenta après coup d'exercer des pressions sur la police pour que l'inculpé soit relâché(21).

Bénazir Bhutto, lors de son accession au pouvoir, n'a été ni capable d'infléchir la politique du Pakistan en Afghanistan domaine réservé des militaires, ni n'a eu la possibilité de s'attaquer profondeur au trafic. Et les quelques mesures prises en ce sens, me l'extradition de deux grands barons de l'héroïne aux États et le projet de confisquer les biens des trafiquants qu'elle s'apprêtait à faire voter peu avant sa chute, ont suffi pour que les militaires participent activement à l'entreprise visant à la déstabiliser avant de la renverser(22).

Les services secrets occidentaux se disent aujourd'hui persuadés qu'une partie de l'équipement de l'armée pakistanaise est payée par des caisses noires alimentées par l'argent de l'héroïne(23). Certains observateurs estiment que cet argent pourrait 
également contribuer à payer la centrale nucléaire dont la vente a été autorisée par le président Mitterrand lors de sa visite au Pakistan en février 1990, alors que Benazir Bhutto était encore au pouvoir.

La nouvelle narco-bourgeoisie

21 Le rôle de l'argent de la drogue ne se limite pas au complexe militaire. Depuis quelques années il pénètre l'ensemble de l'économie. D'une part parce que l'on a assisté durant la dictature à l'émergence d'une bourgeoisie de parvenus, composée précisément d'anciens militaires reconvertis dans les affaires. Elle a été longtemps maintenue à l'écart par la bourgeoisie traditionnelle, en particulier celle de Lahore, qui n'acceptait pas de côtoyer ces personnages, dont la richesse n'avait pas d'origine avouable. Mais le Pakistan souffrant d'une pénurie chronique de devises, a fini par accepter d'associer les narcos-industriels à ses affaires. Cette attitude s'explique dans la mesure où ces militaires sont modernes, concurrents de la bourgeoisie indienne, nationalistes, partisans du programme nucléaire et d'une politique Sud-Sud à tendance islamique.

Ces conditions favorables au développement de l'économie de la drogue ont été encore aggravées par le contexte politique et économique qui règne depuis octobre 1990. Le Premier ministre, Nawaz Sharif, est l'héritier spirituel du général Zia qui a permis la privatisation de son groupe, Ittefaq Industries, un des plus importants du Pakistan, nationalisé à l'époque par Ali Bhutto et qui, de plus, lui a accordé d'importants prêts bancaires. Le fils du général Zia, Ejaz ul-Haq fait partie du ministère. Le Premier ministre est également très lié aux services secrets de l'armée, c'est-à-dire à l'ISI, véritable cheville ouvrière du trafic d'héroïne avec l'Afghanistan. Au Parlement siège dans les rangs de la coalition au pouvoir - l'AIDI- un véritable groupe parlementaire de barons de l'héroïne, au premier rang desquels figure Haji Ayub Zakakhel, un des huit élus des agences tribales. Le statut particulier des ces régions impliquant en particulier que seules les Jirgas des différents clans désignent les députés, cela a facilité la tâche du seigneur de Landikotal qui aurait payé chacun de ses grands électeurs 2500 dollars. A peine élu, et jouissant donc de l'immunité parlementaire, il a donné une conférence de presse à Islamabad pour déclarer que le gouvernement de Bénazir Bhutto l'avait injustement accusé.

23 En outre, ces productions viennent opportunément compenser la pénurie grandissante de devises dont souffre l'économie légale du Pakistan. Avant même que n'éclat la guerre du Golfe, le gouvernement estimait que le montant des envois de devises par les travailleurs émigrés - $10 \%$ de la population active pakistanaise travaille à l'étranger après avoir atteint trois milliards de dollars en 1982, et représenté encore 1,8 milliard de dollars en 1989, n'aurait de toute façon pas dépassé 400 millions en 1990. Il n'a pas dépassé en fait 150 millions de dollars à la suite des départs Koweït. Cette perte sèche de ressources sera encore aggravée par tentation de la facture pétrolière de 200 millions de dollars à la suite des départs du fait de l'interruption des exportations du Koweït qui consentait des tarifs préférentiels au Pakistan.

D'autre part, les Etats-Unis, convaincus que ce pays cherche à doter de l'armement nucléaire, ont suspendu depuis le 1er octobre une aide économique et militaire qui s'élevait à 600 millions dollars, et même si celle-ci vient à reprendre, ce sera à un niveau très inférieur.

25 L'attitude des Etats-Unis pourrait donc entraîner un durcissement de la position des créanciers du Pakistan comme la Banque mondiale, le FMI et les membres du Club de Paris. Le gouvernement de Nawaz Sharif prévoit en 1991 un déficit du budget atteint 
deux milliards de dollars. Celui-ci sera provoqué en particulier des dépenses militaires qui représentent $39,1 \%$ du budget environ du PNB. Le service d'une dette extérieure qui passe 15 milliards de dollars sera de 3,2 milliards de dollars, soit 42,3\% des ressources en devises.

Certes, cédant aux pressions américaines, Nawaz Sharif a pris un certain nombre de mesures contre le trafic en 1991. Deux des grands barons de l'héroïne, inculpés sous le gouvernement de Bénazir Bhutto, Iqbal Baig et Anwar Kattak ont été condamnés à 7 ans de prison au mois de juillet. Deux membres de l'Assemblée provinciale et un chef de tribu du Baloutchistan, alliés du pouvoir, dont l'objet d'une enquête dans l'affaire d'une saisie de 1,7 tonne d'héroïne en octobre 1990. Mais dans le même temps les mafias de l'héroïne se montrent de plus en plus audacieuses, comme le montre l'assassinat d'un des chefs des services de renseignement des douanes de Karachi, Salim Malik en juillet 1991(24). Après cette vengeance contre celui qui avait contribué à faire condamner Iqbal Baig et Anwar Kattak et à faire arrêter un autre baron de l'hérö̈ne, Ashraf Rana (que ses amis politique ont fait ensuite libérer), le journaliste Kamran Khan qui avait couvert cette affaire pour l'hebdomadaire News a été blessé d'une douzaine de coups de couteau, le 23 septembre 1991, après avoir reçu des menaces téléphoniques tendant à le dissuader d'écrire des articles "antigouvernementaux". Ainsi, semble-t-il, les familles pakistanaises de la drogue qui jusque-là préféraient agir dans l'ombre sont en train d'adopter les méthodes d'intimidation qui sont le propre des mafias, de Palerme à la Colombie.

\section{RÉSUMÉS}

La spécialisation récente du Pakistan dans la drogue a été provoquée par la rencontre fortuite de divers facteurs : mise en place d'une politique prohibitionniste par le général Zia, révolution iranienne et invasion soviétique en Afghanistan. La production d'opium et sa transformation en héroïne sont localisées dans les zones tribales du nord-ouest du Pakistan dont la spécificité est d'être contrôlée non par le gouvernement central mais par des autorité: locales à base tribale. II ne faudrait pas en déduire que les organisations mafieuses soient cantonnées à ces zones. A l'intérieur du pouvoir et de l'armée, des réseaux existent qui recyclent l'argent de la drogue dont une partie sert semble-t-il à financer secrètement l'équipement militaire et les recherches nucléaires du pays. De plus, le rôle de la drogue ne se limite pas au complexe militaire, une narcobourgeoisie se développe rapidement, investit le pouvoir politique et est en train d'adopter à l'égard de ses adversaires, des méthodes d'intimidation à la colombienne.

\section{INDEX}

Index géographique : Pakistan

Mots-clés : contrebande et trafic, drogue, économie politique 
AUTEUR

ALAIN LABROUSSE

Ecrivain, Président de l'Observatoire géopolitique des drogues 